\title{
Human Embryonic Stem Cells as an In Vitro Model for Human Vascular Development and the Induction of Vascular Differentiation
}

\author{
Sharon Gerecht-Nir, Anna Ziskind, Smadar Cohen, and Joseph Itskovitz-Eldor \\ Biotechnology Interdisciplinary Unit (SG-N) and Faculty of Medicine (AZ, JI-E), Technion - Israel Institute of \\ Technology, Department of Obstetrics and Gynecology (SG-N, AZ, JI-E), Rambam Medical Center, Israel Medical \\ Center, Haifa, Department of Biotechnology Engineering and the Institute for Applied Biosciences (SC), Ben-Gurion \\ University of the Negev, Be'er-Sheva, Israel
}

\begin{abstract}
SUMMARY: Early embryonic blood vessels are typically composed of fragile tubes of endothelial cells encircled by vascular smooth muscle cells. Early human vasculogenesis was explored in spontaneous and directed differentiation models derived from human embryonic stem (HES) cells. In a 3-dimensional (3D) model, HES cells were studied for their potential for vascular differentiation during the spontaneous formation of embryoid bodies. Directed differentiation was investigated by means of a 2-dimensional (2D) differentiation method to promote vascular differentiation from HES cells (without the formation of embryoid bodies). Using this latter approach, up-regulation of early lineage markers of endothelial progenitors were induced. Additional culture under strict conditions and exposure to angiogenic growth factors resulted in a prolonged differentiation pathway into mature endothelial cells and up-regulation of vascular smooth muscle cell markers. The use of 3D collagen gels and Matrigel assays for the induction and inhibition of human vascular sprouting in vitro further established the vascular potential of the cells generated by the 2D differentiation system. Our study shows that HES cells can provide useful models to study early differentiation and development of blood vessels. Moreover, the 2D differentiation model facilitates both the production of vascular lineage cells from HES cells for various potential therapeutic applications and also provides a model for studying the mechanisms involved in early human embryonic blood vessel development. (Lab Invest 2003, 83:1811-1820).
\end{abstract}

$E$ arly in embryonic development, blood vessel formation occurs by a process referred to as vasculogenesis, in which endothelial cell (EC) precursors differentiate, expand, and coalesce to form a network of primitive tubules (Yancopoulos et al, 2000). These blood vessels are typically composed of two main cell types, each serving a different function: the internal ECs line the vascular channels, whereas the external vascular smooth muscle cells (v-SMCs) protect the fragile channels from rupture and control blood flow (Carmeliet, 2000). Previous research in the field of endothelial embryonic cells has dealt with their functions, origin, and nature. Endothelial embryonic cells have been shown to promote liver organogenesis (Matsumoto et al, 2001), induce differentiation of the pancreas (Lammert et al, 2001), and transdifferentiate into cardiac muscle cells under specific conditions (Condorelli et al, 2001). Although the nature of endothelial precursors is not yet fully understood, it is

\section{DOI: 10.1097/01.LAB.0000106502.41391.F0}

Received September 22, 2003.

This work was supported by The Fund for Medical Research and Development of Infrastructure and Health Services, Rambam Medical Center, and the Technion Research and Development Foundation, Ltd.

Address reprint requests to: Dr. Joseph Itskovitz-Eldor, Department of Obstetrics and Gynecology, Rambam Medical Center, P.O. Box 9602, Haifa31096,Israel.E-mail: Itskovitz@rambam.health.gov.il. becoming clear that the generation of $\mathrm{v}-\mathrm{SMC}$ is closely linked to vascular development. The first v-SMCs surrounding the embryonic endothelial tubes were shown to transdifferentiate from the endothelium (Gittenberger-de Groot et al, 1999), and an embryonic common vascular progenitor was later reported to differentiate into ECs and v-SMCs (Yamashita et al, 2000).

Since their first derivation in 1998 (Thomson et al, 1998), it has been shown that human embryonic stem (HES) cells can terminally differentiate into several cell types, including ECs (Levenberg et al, 2002). Spontaneously differentiated ECs were isolated from embryoid bodies (EBs) using platelet endothelial cell adhesion molecule-1 (also known as CD31) as a marker for ECs (Levenberg et al, 2002). These sorted cells exhibited purity of approximately $80 \%$ with endothelial specific marker and showed additional endothelialspecific characteristics, such as vessel-like formation both in vitro and in vivo (Levenberg et al, 2002).

The present study was undertaken in an attempt to analyze HES cells as an in vitro model for human vascular development and further simplify vascular cells derivation for future clinical applications. Spontaneously differentiating human ES cells (ie, human EBs) were examined for specific vascular markers. Both EC and $\mathrm{V}-\mathrm{SMC}$ markers were shown to be present during 3-dimensional (3D) spontaneous in vitro differentiation of HES cells. As type-IV collagen 
was reported to accelerate ES cells toward mesodermal differentiation (Nishikawa et al, 1998; Sone et al, 2003; Yamashita et al, 2000), adjustments for its application to HES cell 2-dimensional (2D) mesodermal differentiation were established and optimized. The underlying hypothesis was that neither co-culture with a specific differentiation-inducible feeder layer nor EB formation are necessary for efficient differentiation of HES cells into vascular-lineage cells. This hypothesis was confirmed when the up-regulation of endothelial progenitor markers was subsequently encouraged, and continuous manipulation of the cells with specific angiogenic factors resulted in EC and V-SMC 2D differentiation. This 2D culture system for mesodermal differentiation also induced sprouting when 3D gel assays were used. The 3D sprouting was inhibited by the addition of antihuman vascular endothelial cadherin (VE-cad) to the culture system. All together, this system facilitates the production of vascular lineage from HES cells and provides a platform for the study of human vasculogenesis and angiogenesis.

\section{Results}

\section{Tracing ECs and v-SMCs in Human EBs}

To explore the early vasculature organization in spontaneously differentiating HES cells, 10- to 15-day-old EBs were examined ( $n=8$; at least 10 EBs were stained in each experiment). CD34 ${ }^{+}$cells in these EBs exhibited strong staining and showed vasculature arrangements along different areas within the EBs (Fig. 1A). Examination of serial sections of EB also revealed depth organization of vessel-like structures formed by $\mathrm{CD} 4^{+}$cells (Fig. 1B, and video supplement available at http://labinvest.uscapjournals.org). Occasionally, scattered CD34 ${ }^{+}$round cells, not in an organized structure, could also be observed (Fig. 1C). These cells are probably CD34 ${ }^{+}$hematopoietic cells, which have been shown to be present during this stage of EB development with a low frequency (Chadwick et al, 2003). However, staining for $\mathrm{CD} 31^{+}$cells was less intense and revealed mostly channel-like structures of grouped cells within the EBs (Fig. 1, D and $\mathrm{E})$. For the identification of V-SMCs in the developing EBs, $\alpha$-smooth muscle actin (SMA), calponin, and smooth muscle myosin heavy chain (SM-MHC) served as markers (Gittenberger-de Groot et al, 1999; Sone et al, 2003). Various $\mathrm{SMA}^{+}$cell types were observed, including epithelial cells organized as sheets along different areas of the EBs, flat cells with intensive actin expression (neither cell-type illustrated), and more elongated cells, which created thick tubes (Fig. 1, F and G). Similar cell morphologies were revealed after staining for calponin (data not shown). Positive SM-MHC cells were rare within the EBs and appeared as flat, mainly elongated cells around obscure voids (Fig. 1H).

\section{D-Induced Mesodermal Differentiation of HES Cells}

Because spontaneously formed human EBs vary extensively (Itskovitz-Eldor et al, 2000), the use of vascular cells derived from HES cells for research and clinical applications necessitates improved and better-controlled differentiation procedures. The model and research strategy (Fig. 2A) described here
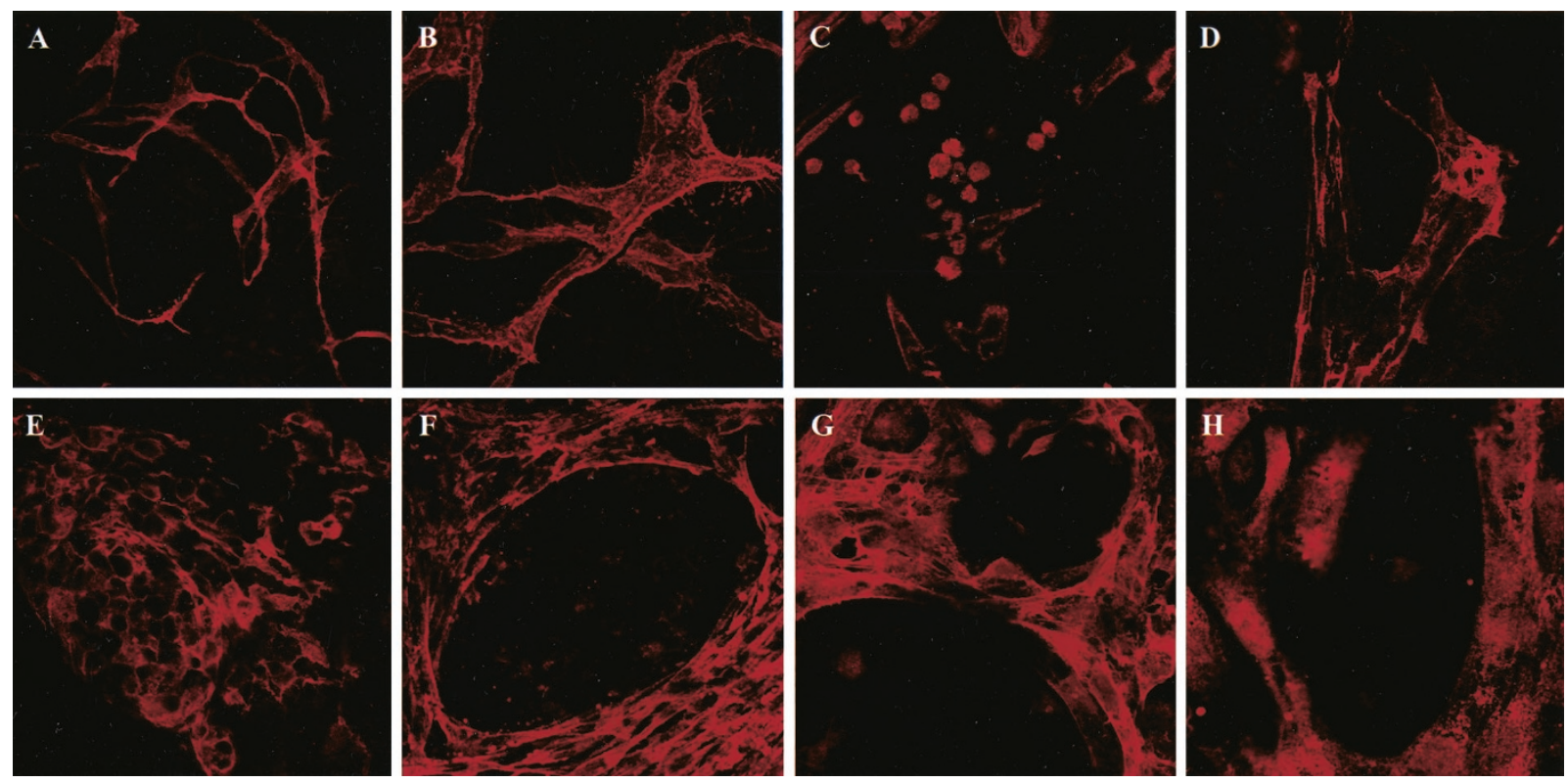

Figure 1.

Spontaneous vascular differentiation in embryoid bodies (EBs). Confocal microscopy of stained 10 - to 15 -day-old human EBs. (A) CD34 ${ }^{+}$cells forming vascular networks along the EBs $(\times 200)$. (B) Projection of $C D 34^{+}$cells serial sections within an EB $(\times 600)$. A video is available at http://labinvest.uscapjournals.org $(\times 600)$. (C) Occasionally round cells are stained with $\mathrm{CD}^{+} 4^{+}(\times 600)$. (D and E) CD31+ cell groups organized in specific channel-like structures $(\times 600)$. $(\mathrm{F})$ Elongated smooth muscle actin $(\mathrm{SMA})^{+}$cells surrounding voids with no detectable network formation $(\times 200)$. (G) $\mathrm{SMA}^{+}$and $(\mathrm{H})$ smooth muscle myosin heavy chain $(\mathrm{SM}-\mathrm{MHC})^{+}$cells surrounding voids, forming flat thick tubes $(\times 600)$. 
A
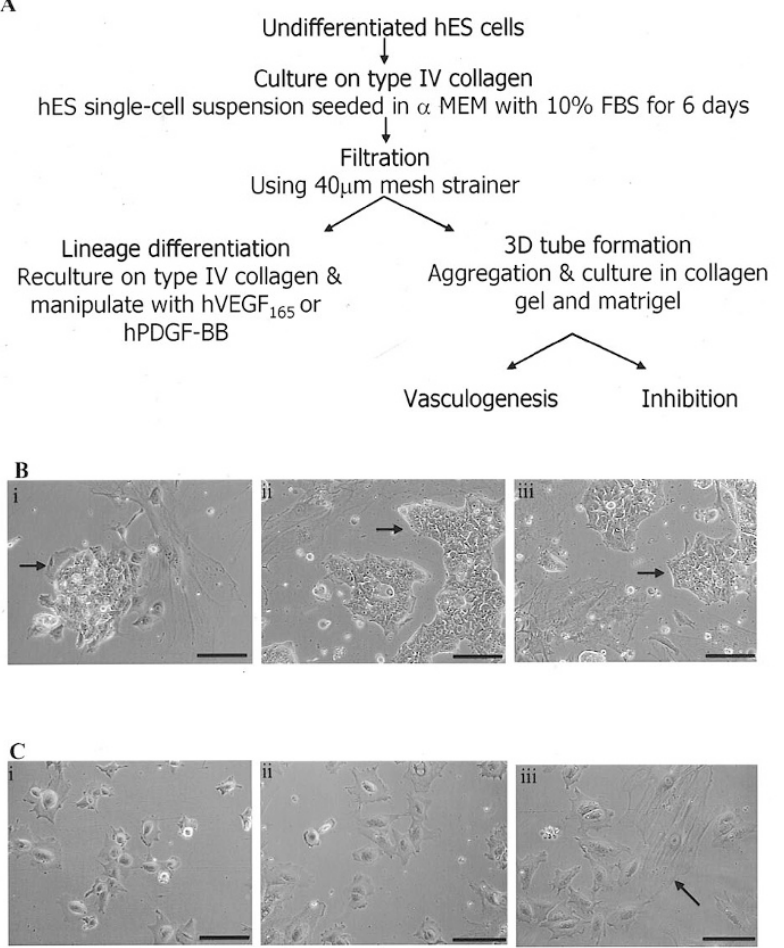

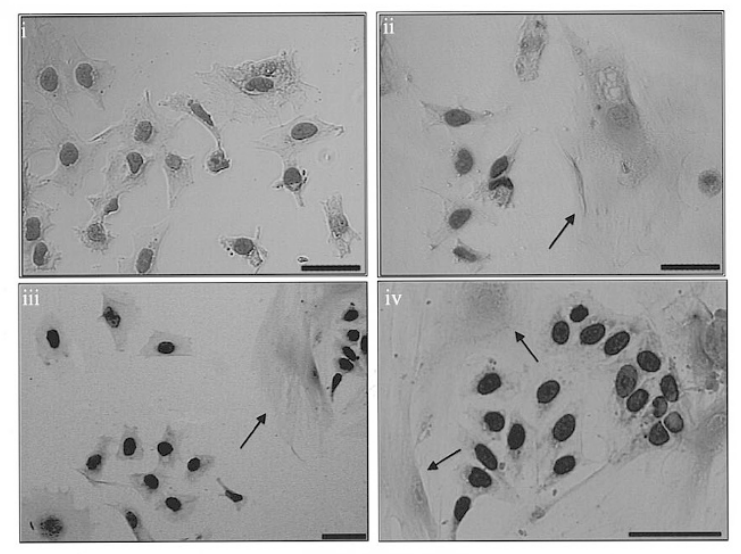

E

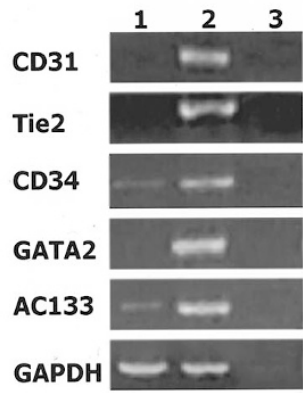

Figure 2.

Two-dimensional (2D) human embryonic stem (HES) cell mesodermal differentiation. (A) Outline of the differentiation procedure. (B) Inverted light microscopy of 6-day-old HES culture of cell aggregates on type-IV collagen shows undifferentiated HES cells (arrows) with different types of differentiated cells. (C) Inverted light microscopy of 6-day-old single-cell suspension cultures shows two cell types: large flat cells with fiber arrangement (arrow) and smaller flat cells with large nuclei. (D) Proliferative ability is demonstrated by 5-bromo-2'-deoxyuridine (BrdU) labeling: (i) most small flat cells were labeled; (ii to iv) large flat cells (arrows) did not incorporate BrdU. (E) Reverse transcription PCR analyses revealed up-regulation of specific endothelial progenitor markers: $1=$ undifferentiated HES cells, $2=$ filtrated 2D differentiated HES cells on type-IV collagen for 6 days, and $3=$ control. Bar $=100 \mu \mathrm{m}$.

relied on the finding that a $3 D$ EB structure is not necessary for the differentiation of lateral mesodermal cells in either mouse (Nishikawa et al, 1998) or primate ES cells (Sone et al, 2003). The former showed use of type-IV collagen-coated dishes as an efficient 2D differentiation model, which promotes lateral mesodermal differentiation (also shown by Nishikawa et al [1998]). Thus, undifferentiated HES cells were grown as previously described (Amit et al, 2000), and then removed from their feeder layers using type-IV collagenase, and plated as aggregates on type-IV collagen-coated dishes in the suggested differentiation medium without any addition of growth factors (Yamashita et al, 2000). Under these culture conditions, undifferentiated colonies could be observed along with several different cell types that spread out from the undifferentiated colonies (Fig. 2B). Therefore, EDTA, PBS, and fetal bovine serum were used for the digestion of the undifferentiated cells, followed by separation into individual cell suspensions. Moreover, according to Yamashita et al (2000), a specific cellseeding concentration is needed to induce intensified endothelial differentiation. Seeding the HES cells at the cell concentration recommended by Yamashita et al $(2000)\left(1 \times 10^{4}\right.$ cells $\left./ \mathrm{cm}^{2}\right)$ resulted in cell death (data not shown). Several 2D differentiation experiments with different cell-seeding concentrations on gelatin or type-IV collagen-coated dishes led to the conclusion that a higher cell-seeding concentration $\left(1 \times 10^{5}\right.$ to $1.5 \times 10^{5}$ cells $/ \mathrm{cm}^{2}$ ) results in undifferentiated colonies surrounded by mesodermally differentiated cells, whereas lower cell-seeding concentrations $\left(5 \times 10^{4}\right.$ to $7 \times 10^{4}$ cells $/ \mathrm{cm}^{2}$ ) encourages the production of a more defined differentiated population on Day 6. Interestingly, this differentiation model produced mainly two types of cell populations: the first consisted of smaller flat cells with large nuclei, and the second consisted of large flat cells with a fiber arrangement (Fig. 2C). Most of the smaller cells incorporated 5-bromo-2'-deoxyuridine (BrdU), indicative of their proliferative state, while the larger cells were not labeled with BrdU (Fig. 2D). Therefore, for continuous differentiation, the resultant cell population was filtered through a $40-\mu \mathrm{m}$ strainer. This filtration stage segregated the large flat cells (retained by the filter) from the small ones (which passed through the filter). Semi-quantitative reverse transcription (RT)-PCR of the filtrated cells revealed an up-regulation of specific endothelial progenitors markers such as CD31, CD34, AC133, Tie2, and Gata2, which were not expressed (or at very low levels) in the undifferentiated HES cells (Fig. 2E). However, v-SMC markers could not be detected in the filtrated cells (data not shown). These results agree with a previous study on HES cells in which these markers were expressed in 6-day-old human EBs (Levenberg et al, 2002). 


\section{Induced Lineage Differentiation}

For the induction of differentiation, the filtrated mesodermally differentiated cells were recultured on type-IV collagen-coated dishes at a lower cell-seeding concentration $\left(2.5 \times 10^{4}\right.$ cells $\left./ \mathrm{cm}^{2}\right)$. The potential for continuous differentiation into ECs was tested in the presence of human vascular endothelial growth factor $(\text { VEGF })_{165}$, an established mitogen of endothelial cells. During reculturing, recognizable vascular arrangements of the maturing cells could be observed, with ring- or cord-like arrangements, depending on the plate coating (Fig. 3, A and B). These conditions resulted in an extensive uptake of Dil-labeled acetylated low-density lipoprotein (Ac-LDL), and also in some of them the presence of von Willebrand factor could be detected (Fig. 3, C and D). Twenty percent of the cells exhibited the endothelial marker CD31 (Fig. 3E). To encourage $\mathrm{v}-\mathrm{SMC}$ differentiation, a known recruitment factor for pericytes platelet-derived growth factor-BB, which is effective in v-SMC differentiation of mouse ES cells (Yamashita et al,
2000) was added to the cultures. After 10 to 12 days, $90 \%$ of the cells expressed $\mathrm{v}$-SMCs markers SMA, calponin, and SM-MHC (Fig. 3F). SMA ${ }^{+}$cells could also be observed in the cultures supplemented with human VEGF $_{165}$.

\section{Tube Formation and Inhibition}

Two 3D culture models were used to study the in vitro vasculogenesis potential of the filtrated-mesodermal cells: type-I collagen gel and Matrigel. These two models are known to promote the formation of $3 D$ vessel-like structures from endothelial cells (Levenberg et al, 2002; Madri et al, 1988). Human VEGF ${ }_{165}$ was added to the culture media to favor endothelial differentiation. Sprouting and network branching could be observed in more than $85 \%$ of the mesodermal cell aggregates $(n=10)$. Transverse sections revealed penetration of the cells into the Matrigel (Fig. 4D), as well as the occasional formation of tube-like structures (Fig. 4E).
A
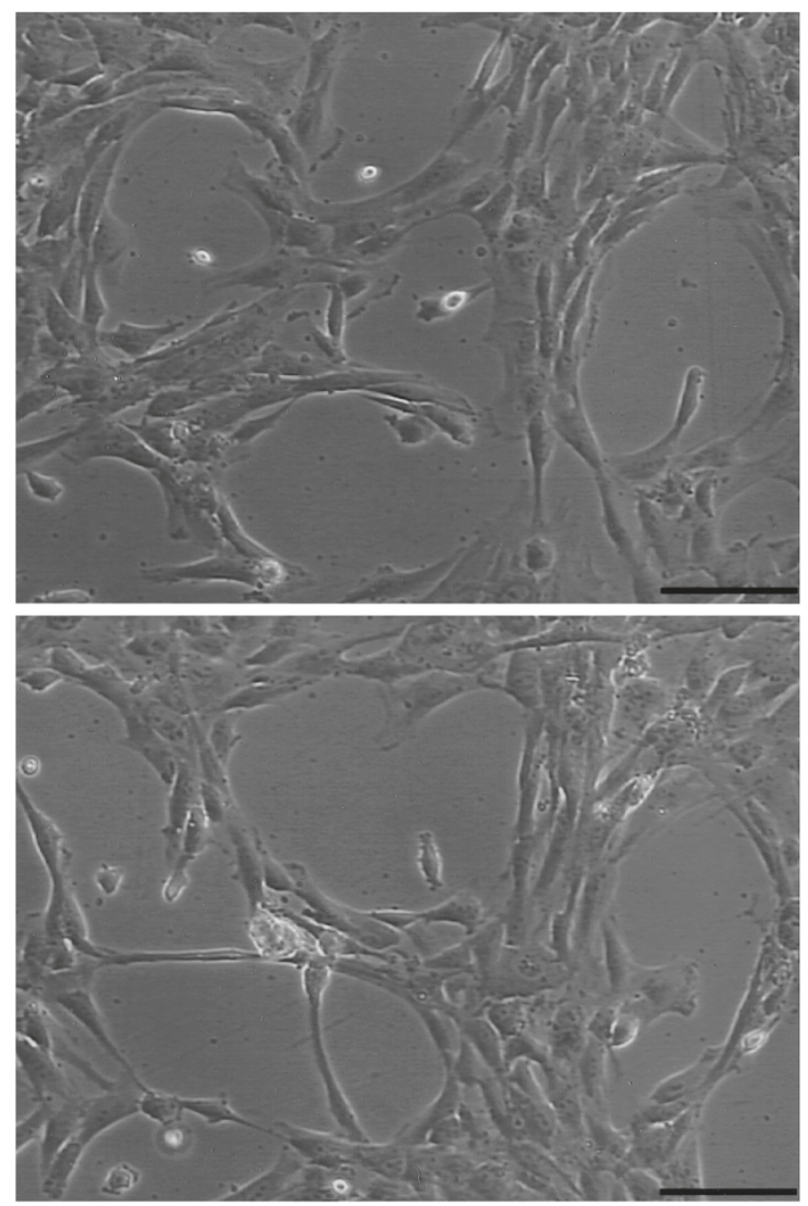

B
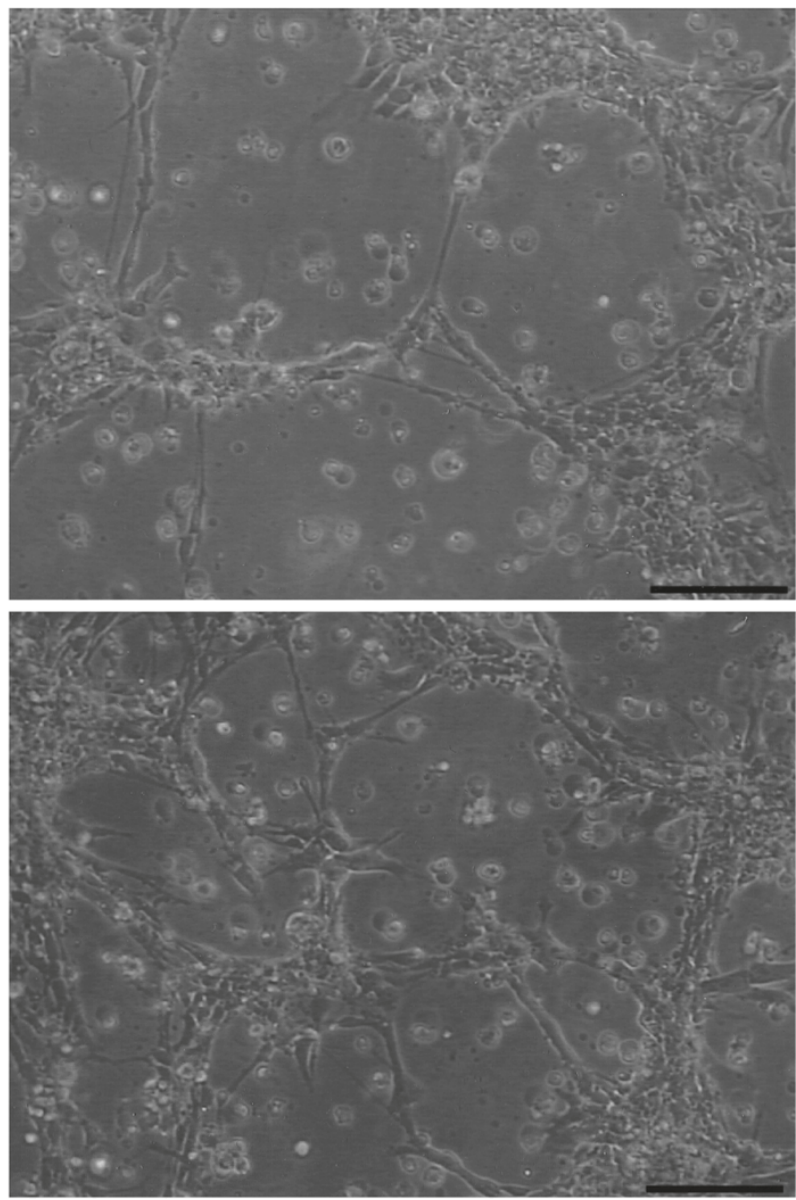

Figure 3.

Lineage differentiation. Filtrated cells recultured with $50 \mathrm{ng} / \mathrm{ml}$ human vascular endothelial growth factor ${ }_{165}$ show (A) ring structures on type-IV collagen and (B) occasional cord-like arrangement on noncoated dishes. (C) Dil-labeled Ac-LDL incorporation (cells in bright-field illumination on the left and in fluorescence illumination on the right). (D) Examination of additional segregated individual cells revealed (i) Dil-labeled Ac-LDL metabolism together with (ii) perinuclear von Willebrand factor, and (iii) co-expression. (E) CD31+ cells. (F) Reculturing with $10 \mathrm{ng} / \mathrm{ml}$ human platelet-derived growth factor-BB for 10 to 12 days resulted in specific vascular smooth muscle cells marker expression: (i) smooth muscle actin, (ii) calponin, (iii) smooth muscle myosin heavy chain, and (iv) mouse IgG as a control. Nuclei stained with Dapi. Bar $=100 \mu \mathrm{m}$. 
C

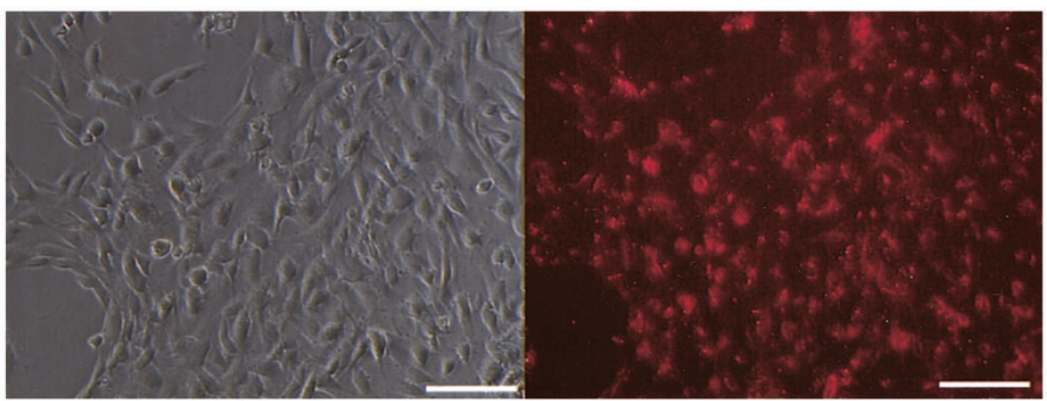

D
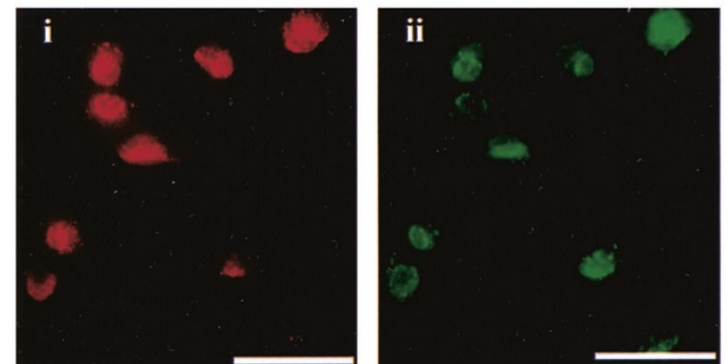

$\mathbf{F}$
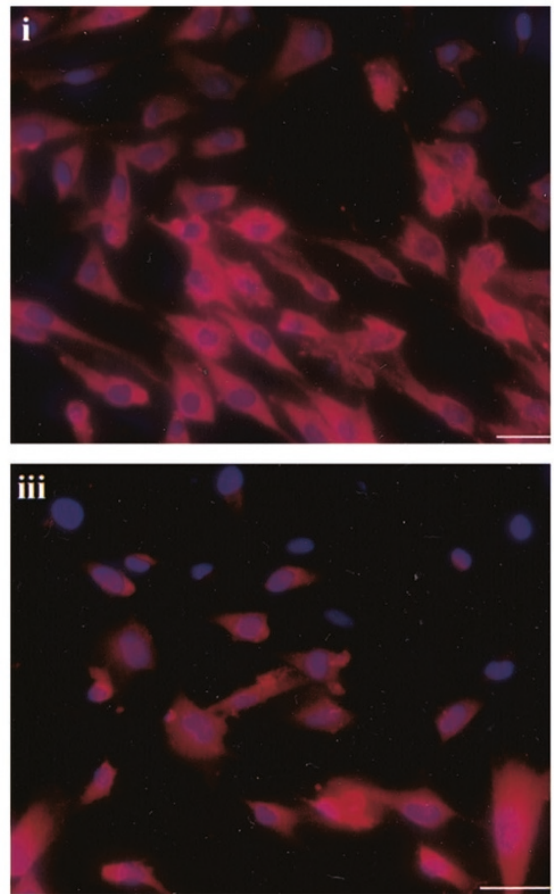

Ultrastructural observation by transmitted electron microscopy also revealed typical tube-like arrangement of elongated ECs within the Matrigel (Fig. 5A), and the presence of Weibel-Palade bodies in the endothelial cell cytoplasm as previously described (Hatzopoulos et al, 1998) (Fig. 5B). By transmitted electron microscopy examination, sporadic apoptotic cells were also observed along the elongated cells, though no other cell types were identified.

Immunological characterization $(n=3)$ of the sprouting revealed that more than $70 \%$ of the cells that penetrated the Matrigel expressed CD34, and only $25 \%$ stained for KDR (VEGFR2). More than $85 \%$ of the tube-like structures formed within the Matrigel
$\mathbf{E}$
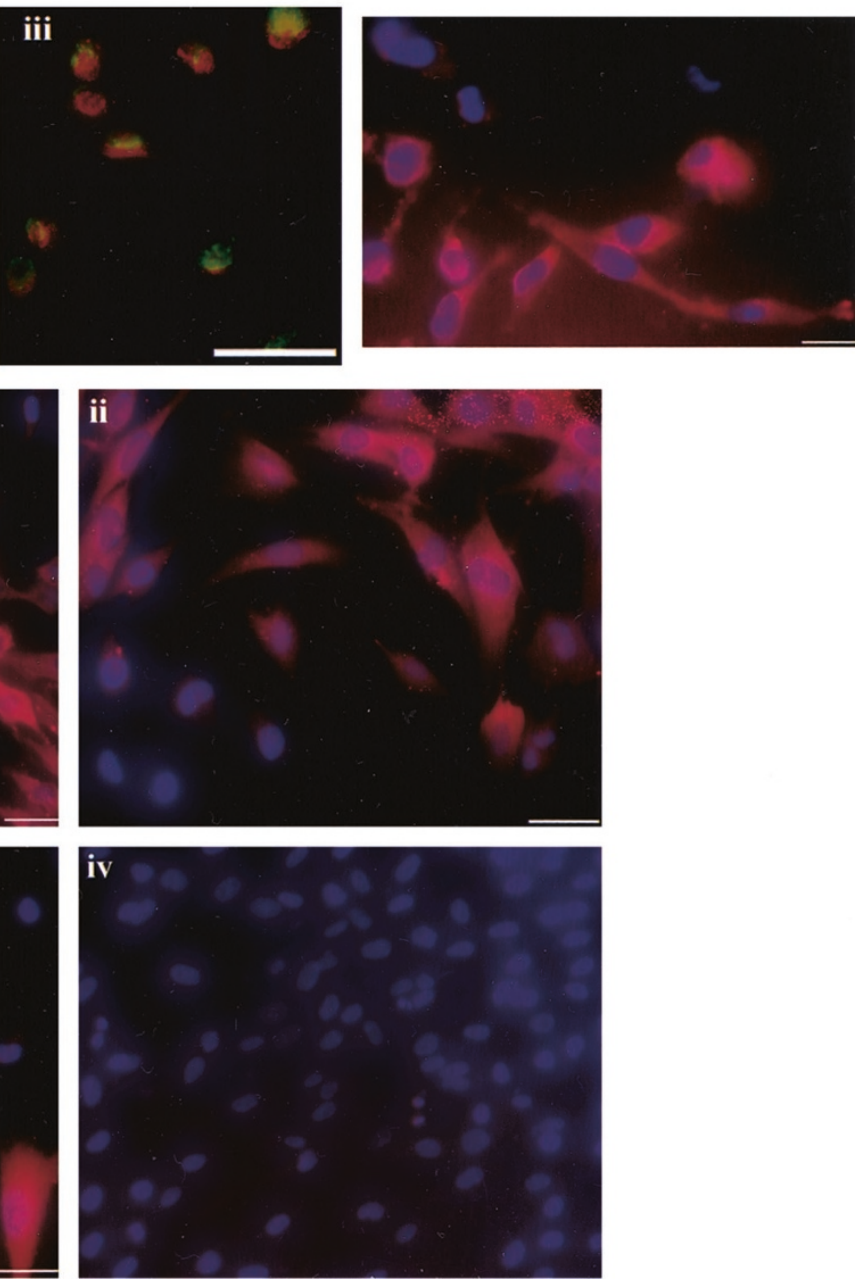

could be easily observed with anti-VE-cad and antiTie2 (Fig. 5, C and D).

The 3D model was also tested for its ability to be used as a tool to investigate the molecular mechanisms of human angiogenesis. It was reported that VE-cad-/- mouse ES-derived EBs failed to develop endothelial sprouts (Feraud et al, 2001). Furthermore, monoclonal anti-VE-cad was suggested to be a potent inhibitor of angiogenesis, tumor growth, and metastasis (Corada et al, 2002; Liao et al, 2000). In addition, different antibodies were shown to affect the modulation of endothelial permeability and in vitro tube formation (Corada et al, 2001, 2002). These observations, along with the newly suggested 


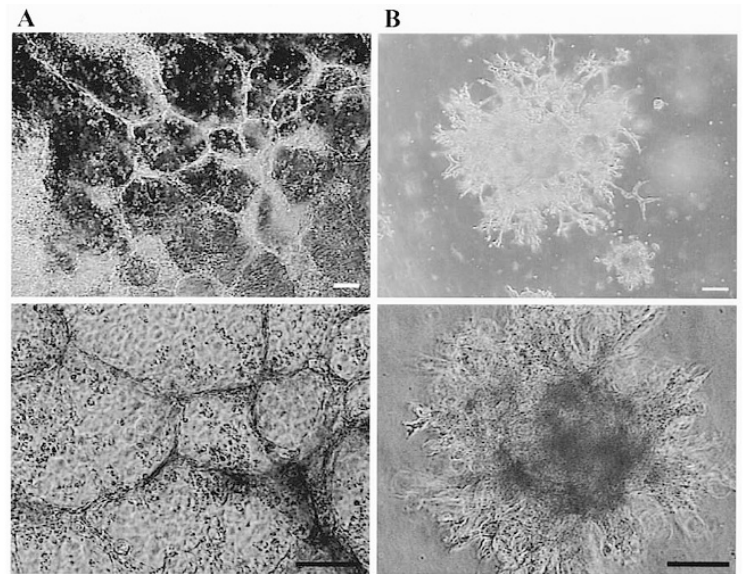

C

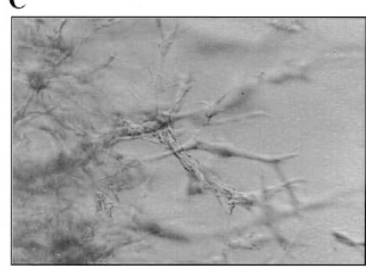

D

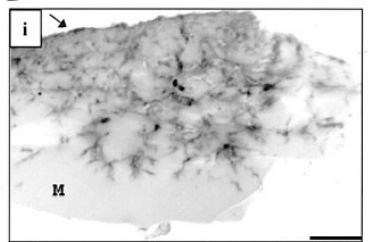

E
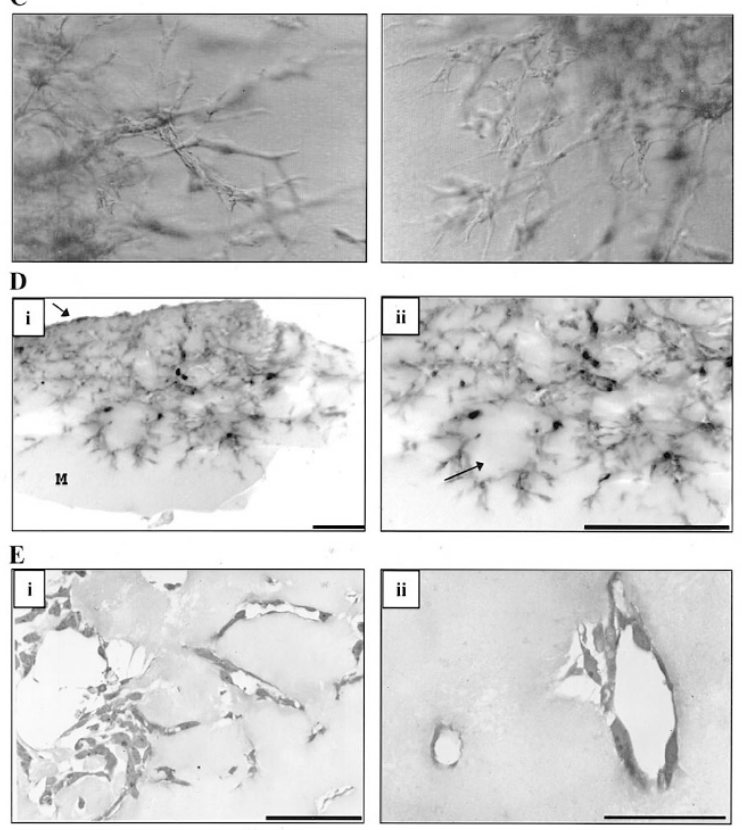

Figure 4.

Sprouting from differentiated human embryonic stem cells. Differentiated cells were allowed to aggregate for 24 hours in medium supplemented with human vascular endothelial growth factor ${ }_{165}$, after which they were seeded into type-I collagen or Matrigel in the same medium. (A) Tube-like formation after 7 days in type-I collagen gel and (B) aggregate sprouting in Matrigel (low and high magnification). (C) Two different areas of 3-dimensional sprouting on Matrigel, documented with Hoffman microscopy. (D) Longitudinal histological sections showed (i) seeded cells (arrow) penetrating into the Matrigel $(M)$ and (ii) forming primitive tube-like structures (arrow) (higher magnification). (E) Transverse histologic sections of the cell-seeded Matrigel exhibited different sizes and shapes of tube-like structures formed within the Matrigel. Bar $=100 \mu \mathrm{m}$.

antibody-based therapeutic model for blocking angiogenesis (Hicklin et al, 2001), encouraged us to test this approach for inhibiting blood vessel formation from HES cells. Using the same assay as previously examined on human endothelial cells (Corada et al, 2001), a specific clone of monoclonal anti-human VE-cad, BV6, was applied to a 3D in vitro vessel formation model $(n=3)$. The differentiated HES cells were tested for their potential use as a model to study the molecular mechanisms and inhibitors of angiogenesis. The addition of monoclonal anti-CD34, a specific clone of anti-VE-cad, as well as isotype matching to the Matrigel and culture medium, was examined. The vasculogenic population was blocked with anti-VEcad, ie, the aggregates that were seeded in Matrigel supplemented with BV6 clone of anti-VE-cad hardly sprouted (only $5 \%$ of the seeded aggregates showed occasional sprouting after 6 days in Matrigel) and failed to form tubes and networks on Matrigel (Fig. 6). This outcome conforms to the important role of VEcad in the process of capillary tube formation of HUVEC (Bach et al, 1998).

\section{Discussion}

The present study was undertaken to evaluate the potential use of HES cells as in vitro models for human vascular development and to further develop simple and practical methods for the enrichment and culture of vascular cells for future therapeutic applications. Human early vasculature expresses known endothelial markers such as CD34 and CD31 (Oberlin et al, 2002). As in the developing human embryo, vascular structures could easily be detected in the developing human EBs by both CD34 and CD31. Early v-SMCs markers include SMA, which is a well-known marker for early embryonic v-SMCs, as well as SMA and calponin, which are established markers for more mature embryonic SMCs (Gittenberger-de Groot et al, 1999). Expression of V-SMCs in the human EBs revealed thick tubes and voids whereas no network structures formed by SMA, calponin, or SM-MHC ${ }^{+}$ cells could be detected. Altogether in this study, spontaneously differentiated human EBs were shown to recapitulate the early EC and v-SMC differentiation kinetics occurring during human vasculature development and therefore may serve as a tool to study early events in human embryonic vasculogenesis.

In an attempt to achieve manipulated differentiation, which may yield an increased proportion of vascular cells generated from HES cells, a 2D cultureconditioning environment for directing HES cell differentiation was applied. This approach is based on three important factors: 1) culturing the differentiating HES cells as single cells to avoid any aggregation or EB formation, 2) using a specific cell-seeding concentration and differentiation medium, and 3) using type-IV collagen matrix-based adherent selection. These three factors together with the filtration stage enabled the direction and enrichment of vascular cells without the use of EBs, feeder layers, exogenous agents, or fluorescencebased sorting procedures. Exposure of the 2D differentiating cells to the angiogenic growth factors VEGF and platelet-derived growth factor-BB, up-regulated EC and $\mathrm{V}-\mathrm{SMC}$ markers. These results of HES cell differentiation using a 2D model correspond to recently published results on primate ES cells (Sone et al, 2003).

One of the important advantages of vascular cells is their ability to maintain high proliferative activity, thus rendering them a potential source for building functional human vessels, ex vivo and in vivo. Such an application was recently shown for endothelial progenitor cells harvested from peripheral blood of sheep 

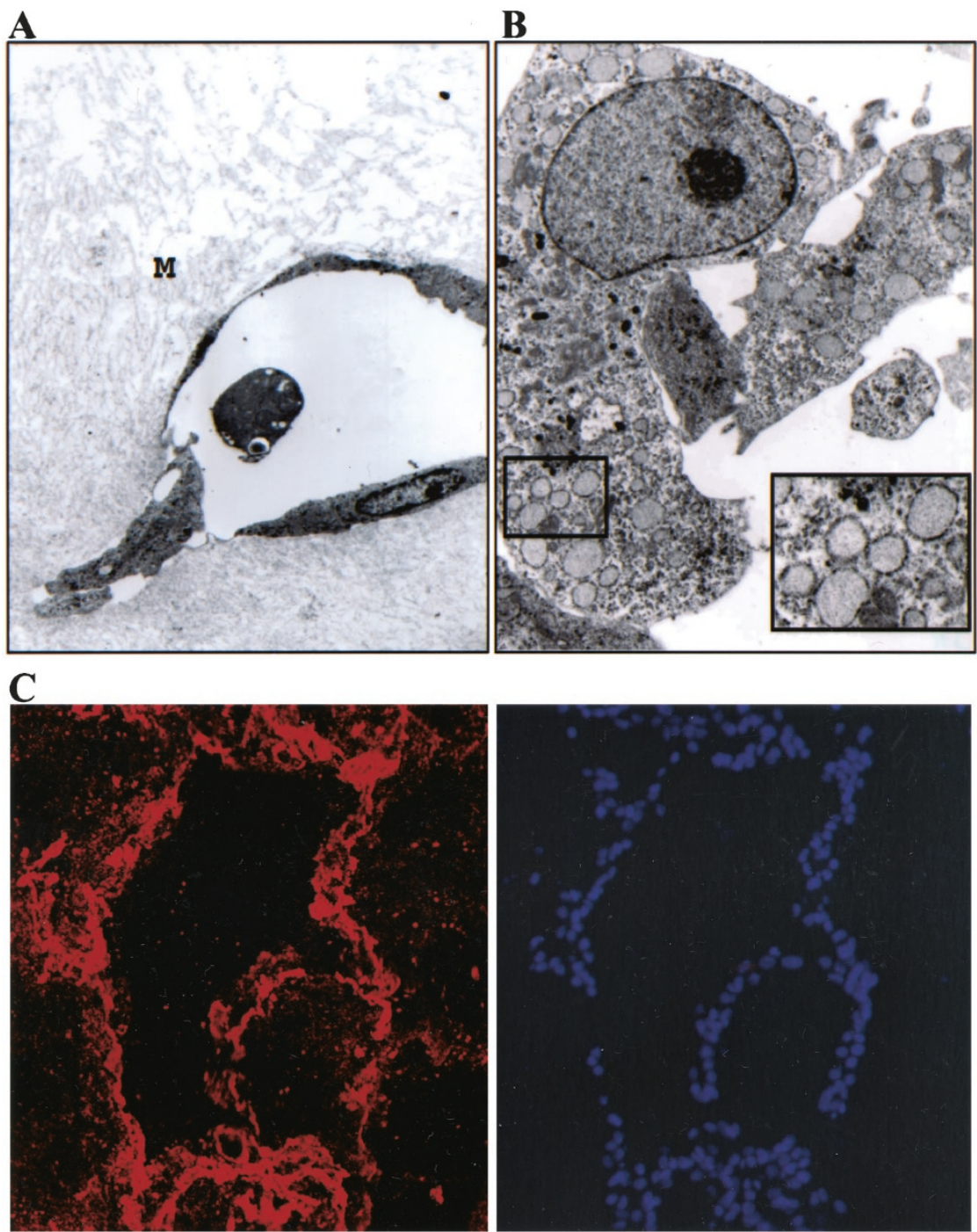

D
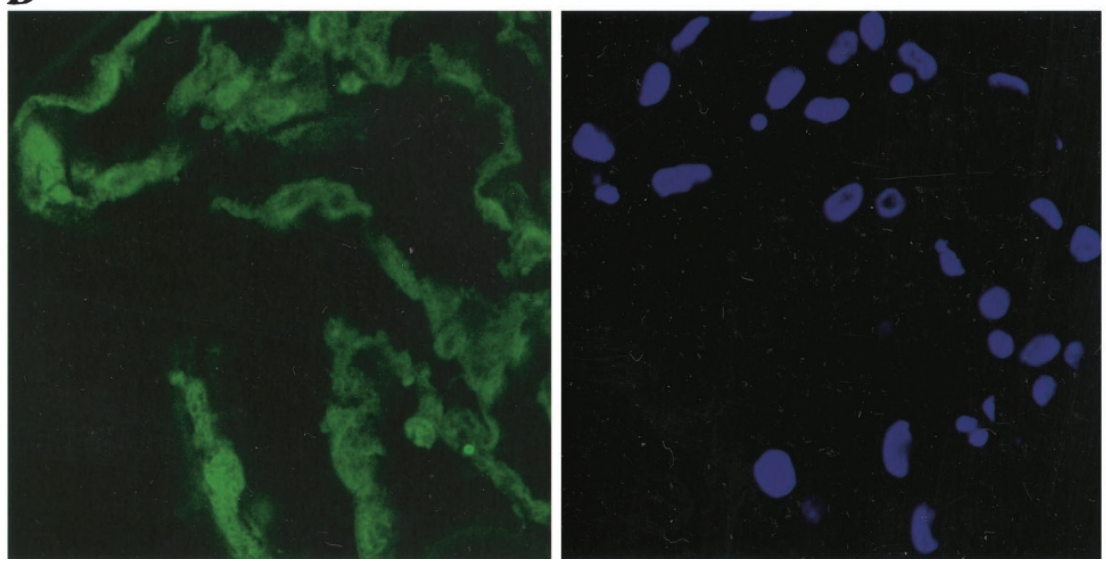

\section{Figure 5.}

Sprouting characterization. (A) Transmitted electron microscopy examination revealed a typical endothelial tube-formation within the Matrigel (M). (B) Weibel-Palade bodies in a single endothelial cell $(\times 6000)$ and detailed in small image $(\times 12,000)$. (C) Vascular endothelial cadherin-positive cells (red; nucleus in blue) could be easily observed in lateral sections, penetrating the Matrigel and forming vessel-like structures $(\times 200)$, as well as (D) Tie2 ${ }^{+}$cells $(\times 600)$.

and seeded on decellularized porcine iliac vessels (Kaushal et al, 2001). Recently, genetically manipulated proliferating human SMCs were reported to be used to engineer mechanically robust human vessels, a vital step toward creating arteries of clinical value for bypass surgery (McKee et al, 2003).

In another application, HES cells can be used as an in vitro research model to examine the vasculogenesis 


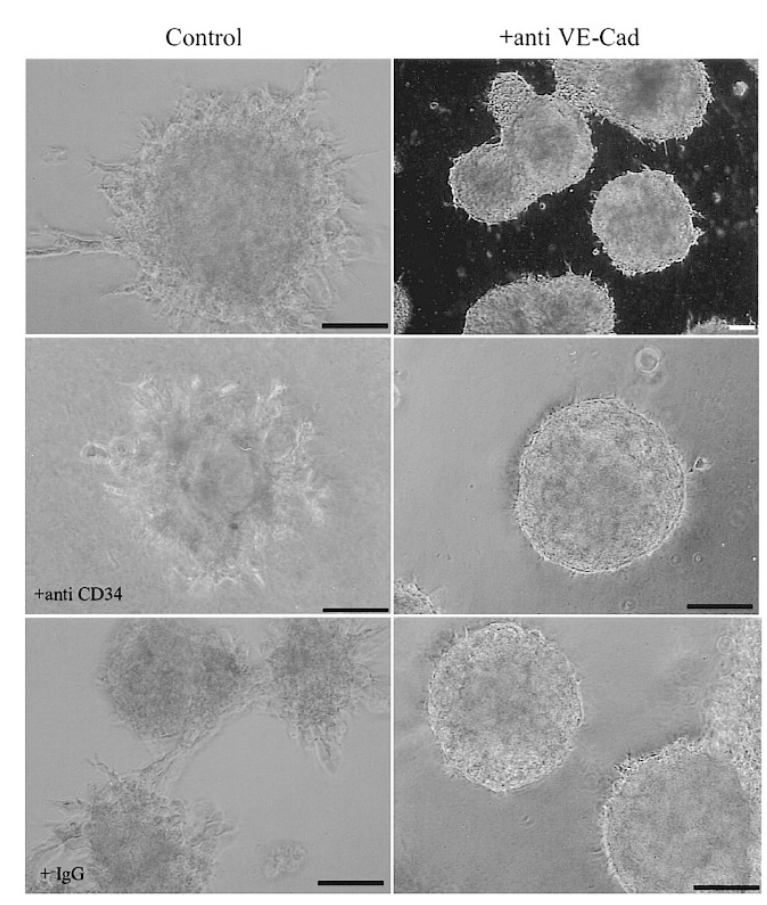

Figure 6.

Sprouting inhibition. Left column: the upper panel shows aggregates seeded on Matrigel, the middle panel shows aggregates seeded on Matrigel supplemented with $50 \mu \mathrm{g} / \mathrm{ml}$ monoclonal anti-CD34, and the bottom panel shows aggregates seeded on Matrigel supplemented with isotype matching. Right column: all panels show aggregates seeded for 7 days on Matrigel with supplementation of $50 \mu \mathrm{g} / \mathrm{ml}$ monoclonal anti-vascular endothelial cadherin, clone BV6, which blocks angiogenesis (right). Bar $=100 \mu \mathrm{m}$.

and angiogenesis mechanisms. Although the complex events leading to angiogenesis are not yet completely understood, considerable interest is currently centered on the inhibition of new vascular growth to treat the spread of cancer. There is considerable need for a suitable in vitro model system to study antiangiogenesis agents. Studies on mouse ES cells showed that spontaneous differentiation via EB formation recapitulates angiogenic events and therefore may be used for angiogenesis research (Feraud et al, 2001; Wartenberg et al, 1998). Hence, HES cells may serve as an in vitro research model to examine the molecular mechanisms involved in human angiogenesis. Evidence of the key role of VE-cad in tube formation (Bach et al, 1998; Feraud et al, 2001) led to the examination of the angiogenic blocking potential of VE-cad using a 3D sprouting model of endothelial progenitor cell populations derived from HES cells. A specific clone of anti-VE-cad, BV6, was able to block $95 \%$ of the sprouting events. These findings are consistent with others (Corada et al, 2001) in which antibodies directed at extracellular domains EC3 affected ECs vascular structure formation. It should be noted that during these experiments high concentrations of antibody were used (50 $\mu \mathrm{g} / \mathrm{ml})$. The reproducibility of the inhibitory effects of anti-VE-cad should be further studied in respect to dose-response effects.

Taking into consideration the limited means available for studying human vasculogenesis and embryonic development, HES cell systems offer the initial steps to- ward understanding these complex and intriguing fields. Further studies using genetic manipulation and singlecell techniques of HES cells and their derivatives are still needed to identify fully and to characterize vascular progenitors and their development.

\section{Materials and Methods}

\section{HES Cell Culture}

Undifferentiating HES cells (H9.2 passages $29+36$ to $29+60$; $\mathrm{H} 13$ passages 31 to 57 ; 16 passages 35 to 50 ; 19 passages 29 to 37 ) were grown on an inactivated mouse embryonic feeder layer as previously described (Amit et al, 2000). They were then separated using type-IV collagenase, resulting in small aggregates. For EB formation, a half confluent six-well plate $\left(60 \mathrm{~cm}^{2}\right)$ of undifferentiated HES cells was used. ES cells were removed from the feeder layers using 1 $\mathrm{mg} / \mathrm{ml}$ type-IV collagenase, further dissociated into small clumps using 1000- $\mu$ l Gilson pipette tips, and cultured in suspension in 50-mm nonadherent Petri dishes (Ein-Shemer, Israel). EBs were grown in medium consisting of $80 \%$ KO-DMEM (Gibco-BRL), supplemented with $20 \%$ defined fetal bovine serum (HyClone), $1 \mathrm{~mm}$ L-glutamine, and 1\% nonessential amino acid stock (all from Gibco-BRL). For individual cell suspension (ie, culture for differentiation studies), HES cells were digested with EDTA $5 \mathrm{~mm}$ in PBS, supplemented with 1\% (v/v) FBS (HyClone), and separated into individual cell suspension using a $40-\mu \mathrm{m}$ mesh strainer (Falcon). Undifferentiated HES individual cell suspensions were plated on type-IV collagen-coated six-well dishes (Becton Dickinson). The cells were seeded at a different density indicated above, in a differentiation medium composed of alpha MEM medium (Gibco-BRL) supplemented with 10\% FBS (HyClone) and $0.1 \mathrm{~mm} \beta$-mercaptoethanol (Gibco-BRL). On Day 6 of culture, the resultant differentiated cells were filtered through a $40-\mu \mathrm{m}$ mesh strainer (Falcon) and analyzed or recultured for differentiation. For reculturing, $2.5 \times 10^{4}$ cells $/ \mathrm{cm}^{2}$ on type-IV collagencoated dishes (Becton Dickinson) were plated in a differentiation medium containing human VEGF $_{165} 50$ $\mathrm{ng} / \mathrm{ml}$ or human platelet-derived growth factor-BB 10 $\mathrm{ng} / \mathrm{ml}$ (R\&D Systems, Inc.) for 10 to 12 additional days.

\section{Collagen Gel and Matrigel Assays}

Six-day-old 2D differentiated and filtrated cells were harvested with EDTA, PBS, and fetal bovine serum. The cell suspension, 0.3 to $0.5 \times 10^{6} \mathrm{cells} / \mathrm{ml}$, was incubated in differentiation medium containing 50 $\mathrm{ng} / \mathrm{ml} \mathrm{VEGF}_{165}$ on uncoated Petri dishes (Ein-Shemer, Israel) for a maximum of 24 hours to induce cell aggregation. For the collagen gel assay, cell aggregates were resuspended in $\times 2$ differentiation medium and mixed with an isovolume of collagen type-I from a rat's tail ( $3 \mathrm{mg}^{*} \mathrm{ml}^{-1}$ ) (Roche). $250 \mu$ l of the mixture was plated in 24-well dishes, maintained at $37^{\circ} \mathrm{C}$ for 15 minutes to allow gelation, and then supplemented with $500 \mu \mathrm{l}$ of differentiation medium containing VEGF $_{165}$ as described above. For the Matrigel assay, 
24-well dishes were coated with $380 \mu$ l of Matrigel (Becton Dickinson) and incubated for 30 minutes at $37^{\circ} \mathrm{C}$. The cell aggregates were seeded on the Matrigel layer in differentiation medium with human VEGF (50 $\mathrm{ng} \mathrm{ml}^{-1}$ ) (R\&D Systems). In all assays, the cells were incubated for 7 to 12 days and analyzed using a phase contrast microscope (Olympus). For angiogenesis inhibition assay, cell aggregates were resuspended in Matrigel before solidification, incubated for 30 minutes at $37^{\circ} \mathrm{C}$, and then differentiation medium was added as described above. To induce inhibition, $50 \mu \mathrm{g} / \mathrm{ml}$ monoclonal VE-cad, clone BV6 (Chemicon) or $50 \mu \mathrm{g} / \mathrm{ml}$ monoclonal CD34 class II (Dako), or 50 $\mu \mathrm{g} / \mathrm{ml}$ mouse IgG (R\&D Systems) were added to the Matrigel culture system. Sprouting was scored among 50 aggregates in each culture system, in at least three independent experiments.

\section{Immunophenotype, Confocal Microscopy, Dil-Labeled Ac-LDL Uptake, and BrdU Incorporation}

EBs were seeded overnight on slides precoated with gelatin. Attached EBs, 2D differentiating cells, and Matrigel-sprouting aggregates were fixed in situ with 4\% paraformaldehyde (Sigma) in PBS (Gibco-BRL) for 30 minutes at room temperature. After blocking with $3 \%$ serum, the cells were stained with one of the following primary antibodies: antihuman CD31, antihuman CD34, antihuman SMA, antihuman calponin, antihuman SM-MHC (all from Dako), antihuman von Willebrand factor (Sigma), antihuman Tie2 (Chemicon), and anti-VE-cad (Santa Cruz Biochemicals). Cells were then rinsed and incubated for 30 minutes with a suitable FITC-conjugated or Cy3-conjugated secondary antibody (Sigma). Dapi (Sigma) or DRAQ5 (Alexis) was added (1:500) to the last rinse. IgG isotypematching served as control, including mouse, goat, and rabbit (all from R\&D Systems). The immunolabeled cells were examined using a fluorescence microscope (Zeiss) or confocal laser scanning system (Bio-Rad Laboratories Ltd.). To study Dil-labeled AcLDL uptake, the cells were incubated with $10 \mu \mathrm{g} / \mathrm{ml}$ Dil-labeled Ac-LDL (Biomedical Technologies, Inc.) for 4 hours at $37^{\circ} \mathrm{C}$. Following incubation, the cells were rinsed three times with $\mathrm{PBS}$, fixed with $4 \%$ paraformaldehyde for 30 minutes, and visualized using a fluorescence microscope (Olympus).

Proliferative capability of the 2D differentiated cells was examined via BrdU labeling. BrdU staining kit (Zymed) was used in situ, according to manufacturer's instructions. In brief, epithelial colonies were incubated with BrdU labeling reagent (diluted 1:100) overnight, washed several times with PBS (Gibco), and fixed with $70 \%$ ethanol for 30 minutes at $4^{\circ} \mathrm{C}$. A standard staining procedure was performed and cells were examined using light microscopy (Olympus).

\section{Histology and Immunohistochemistry}

For histological analyses, gels seeded with cells were fixed in $10 \%$ neutral-buffered formalin, dehydrated in graduated alcohols (70\% to $100 \%$ ), and embedded in paraffin. For general histomorphology 1- to 8- $\mu \mathrm{m}$ sections were stained with hematoxylin/eosin or toluidine blue. Immunofluorescence staining was performed after paraffin removal, with specific antibodies as indicated above. The immuno-labeled cells were examined using a confocal laser scanning system (Bio-Rad Laboratories Ltd.)

\section{Transmitted Electron Microscopy}

Cells seeded in Matrigel or collagen gel were fixed for 1 hour in $3 \%$ glutaraldehyde in $0.1 \mathrm{M}$ sodium cacodylate, post-fixed in 1\% OsO4 in veronal-acetate buffer for 1 hour, and embedded in Epon. Sections (60 to 90 $\mathrm{nm}$ ) cut on an ultramicrotome were mounted on uncoated copper grids, contrast-stained with uranyl acetate and lead-citrate, and then examined with a JEM-100SX (JEOL, Tokyo, Japan) transmission electron microscope at $80 \mathrm{kV}$.

\section{RT-PCR Analysis}

Total RNA was extracted using TriReagent (Sigma) according to manufacturer's instructions. Total RNA was quantified by a UV spectrophotometer, and $1 \mu \mathrm{g}$ was used for each RT sample. RNA was reversed transcripted with M-MLV (Promega) and oligo (dT) primers (Promega) according to manufacturer's instructions. PCRs were done with BIOTAQ DNA Polymerase (Bioline) using $1 \mu \mathrm{l}$ of $\mathrm{RT}$ product per reaction, according to manufacturer's instructions. In some cases $\mathrm{MgCl}_{2}$ concentration (normally $1.5 \mathrm{mmol}$ ) was calibrated (indicated below). To ensure semiquantitative results of the RT-PCR assays, the number of PCR cycles for each set of primers was verified to be in the linear range of the amplification. In addition, all RNA samples were adjusted to yield equal amplification of GAPDH as an internal standard. PCR conditions consisted of the following: 5 minutes at $94^{\circ} \mathrm{C}$ (hot start), 30 to 40 cycles (actual number noted below); $94^{\circ} \mathrm{C}$ for 30 seconds, annealing temperature ( $\mathrm{Ta}$, noted below) for 30 seconds; $72^{\circ} \mathrm{C}$ for $30 \mathrm{sec}-$ onds. A final 7 minutes extension at $72^{\circ} \mathrm{C}$ was performed at the end. The amplified products were separated on $2 \%$ agarose gels with ethidium bromide. Oligonucleotide-specific conditions were as follows: GATA2, 35 cycles, Ta 55 ${ }^{\circ} \mathrm{C}$; AC133, 32 cycles, Ta $60^{\circ} \mathrm{C}$; Tie2, 35 cycles, Ta $60^{\circ} \mathrm{C}$; CD31, 32 cycles $\mathrm{Ta}$ $60^{\circ} \mathrm{C}$; CD 3435 cycles Ta $60^{\circ} \mathrm{C}, \mathrm{GAPDH}, 32$ cycles, Ta $60^{\circ} \mathrm{C}$. The following specific oligonucleotide primers were used: GATA2: 5'AGCCGGCACCTGTTGTGCAA3' (242 bp); AC133: 5'CAGTCTGACCAGCGTGAAAA3' and 5'GGCCATCCAAATCTGTCCTA3' (200 bp); Tie2: 5'ATCCCATTTGCAAAGCTTCTGGCTGGC3' and 5'TGTGAAGCGTCTCACAGGTCCAGGATG3'(512 bp); CD31: 5'CAACGAGAAAATGTCAGA3' and 5'GGAGCCTTCCGTTCTAGAGT3' (260 bp); CD34: 5' TGAAGCCTAGCCTGTCACCT3' and 5'CGCACAGCTGGAGGTCTTAT3' (200bp) GAPDH: 5'AGCCACATCGCTCAGACACC3' and 5' GTACTCAGCGGCCAGCATCG3' (302 bp). 


\section{Acknowledgements}

We thank Professor Raymond Coleman for enthusiastic guidance, assistance, and critical reading of the manuscript; Drs. Hanna Segev and Bettina Fishman for consultation; Michal Amit for culture instruction; Drs. Ofer Shenker and Edith Suss-Toby for confocal microscopy assistance; Sivan Osenberg and Ludmilla Mazor for technical assistance; and Hadas O’Neill for editing.

\section{References}

Amit M, Carpenter MK, Inokuma MS, Chiu CP, Harris CP, Waknitz MA, Itskovitz-Eldor J, and Thomson JA (2000). Clonally derived human embryonic stem cell lines maintain pluripotency and proliferative potential for prolong periods of culture. Dev Biol 227:271-278.

Bach TL, Barsigian C, Chalupowicz DG, Busler D, Yaen CH, Grant DS, and Martinez J (1998). VE-cadherin mediates endothelial cell capillary tube formation in fibrin and collagen gels. Exp Cell Res 238:324-334.

Carmeliet P (2000). Mechanisms of angiogenesis and arteriogenesis. Nat Med 6:389-395.

Chadwick K, Wang L, Li L, Menendez P, Murdoch B, Rouleau A, Bhatia M (2003). Cytokines and BMP-4 promote hematopoietic differentiation of human embryonic stem cells. Blood 102:906-915.

Condorelli G, Borello U, De Angelis L, Latronico M, Sirabella D, Coletta M, Galli R, Balconi G, Follenzi A, Frati G, Cusella De Angelis MG, Gioglio L, Amuchastegui S, Adorini L, Naldini L, Vescovi A, Dejana E, and Cossu G (2001). Cardiomyocytes induce endothelial cells to transdifferentiate into cardiac muscle: Implications for myocardium regeneration. Proc Natl Acad Sci USA 98:10733-10738.

Corada M, Liao F, Lindgren M, Lampugnani MG, Breviario F, Frank R, Muller WA, Hicklin DJ, Bohlen P, and Dejana E (2001). Monoclonal antibodies directed to different regions of vascular endothelial cadherin extracellular domain affect adhesion and clustering of the protein and modulate endothelial permeability. Blood 97:1679-1684.

Corada M, Zanetta L, Orsenigo F, Breviario F, Lampugnani MG, Bernasconi S, Liao F, Hicklin DJ, Bohlen P, and Dejana E (2002). A monoclonal antibody to vascular endothelialcadherin inhibits tumor angiogenesis without side effects on endothelial permeability. Blood 100:905-911.

Feraud O, Cao Y, and Vittet D (2001). Embryonic stem cellderived embryoid bodies development in collagen gels recapitulates sprouting angiogenesis. Lab Invest 81:1669-1681.

Gittenberger-de Groot AC, DeRuiter MC, Bergwerff M, and Poelmann RE (1999). Smooth muscle cell origin and its relation to heterogeneity in development and disease. Arterioscler Thromb Vasc Biol 19:1589-1594.

Hatzopoulos AK, Folkman J, Vasile E, Eiselen GK, and Rosenberg RD (1998). Isolation and characterization of endothelial progenitor cells from mouse embryos. Development 125:1457-1468.

Hicklin DJ, Witte L, Zhu Z, Liao F, Wu Y, Li Y, and Bohlen P (2001). Monoclonal antibody strategies to block angiogenesis. Drug Discov Today 6:517-528.
Itskovitz-Eldor J, Schuldiner M, Karsenti D, Eden A, Yanuka O, Amit M, Soreq H, and Benvenisty N (2000). Differentiation of human embryonic stem cells into embryoid bodies compromising the three embryonic germ layers. Mol Med 6:88-95.

Kaushal S, Amiel GE, Guleserian KJ, Shapira OM, Perry T, Sutherland FW, Rabkin E, Moran AM, Schoen FJ, Atala A, Soker S, Bischoff J, and Mayer JE Jr. (2001). Functional small-diameter neovessels created using endothelial progenitor cells expanded ex vivo. Nat Med 7:1035-1040.

Lammert E, Cleaver O, and Melton D (2001). Induction of pancreatic differentiation by signals from blood vessels. Science 294:564-567.

Levenberg S, Golub JS, Amit M, Itskovits-Eldor J, and Langer $R$ (2002). Endothelial cells derived from human embryonic stem cells. Proc Natl Acad Sci USA 99:4391-4396.

Liao F, Li Y, O’Connor W, Zanetta L, Bassi R, Santiago A, Overholser J, Hooper A, Mignatti P, Dejana E, Hicklin DJ, and Bohlen P (2000). Monoclonal antibody to vascular endothelial-cadherin is a potent inhibitor of angiogenesis, tumor growth, and metastasis. Cancer Res 60:6805-6810.

Madri JA, Pratt BM, and Tucker AM (1988). Phenotypic modulation of endothelial cells by transforming growth factor-beta depends upon the composition and organization of the extracellular matrix. J Cell Biol 106:1375-1384.

Matsumoto K, Yoshitomi H, Rossant J, and Zaret KS (2001). Liver organogenesis promoted by endothelial cells prior to vascular function. Science 294:559-563.

McKee JA, Banik SS, Boyer MJ, Hamad NM, Lawson JH, Niklason LE, Counter CM (2003). Human arteries engineered in vitro. EMBO Rep 4:633-638.

Nishikawa SI, Nishikawa S, Hirashima M, Matsuyoshi N, and Kodama $\mathrm{H}$ (1998). Progressive lineage analysis by cell sorting and culture identifies FLK+VE-cadherin + cells at a diverging point of endothelial and hematopoietic lineages. Development 125:1747-1757.

Oberlin E, Tavian M, Blazsek I, and Peault B (2002). Bloodforming potential of vascular endothelium in the human embryo. Development 129:4147-4157.

Sone M, Itoh H, Yamashita J, Yurugi-Kobayashi T, Suzuki Y, Kondo Y, Nonoguchi A, Sawada N, Yamahara K, Miyashita K, Park K, Shibuya M, Nito S, Nishikawa S, and Nakao K (2003). Different differentiation kinetics of vascular progenitor cells in primate and mouse embryonic stem cells. Circulation 107: 2085-2088.

Thomson JA, Itskovitz-Eldor J, Shapiro SS, Waknitz MA, Swiergiel JJ, Marshall VS, and Jones JM (1998). Embryonic stem cell lines derived from human blastocysts. Science 282:1145-1147.

Wartenberg M, Gunther J, Hescheler J, and Sauer H (1998). The embryoid body as a novel in vitro assay system for antiangiogenic agents. Lab Invest 78:1301-1314.

Yamashita J, Itoh $\mathrm{H}$, Hirashima M, Ogawa M, Nishikawa S, Yurugi T, Naito M, and Nakao K (2000). Flk1-positive cells derived from embryonic stem cells serve as vascular progenitors. Nature 408:92-96.

Yancopoulos G, Davis S, Gale N, Rudge J, Wiegand S, and Holash J (2000). Vascular specific growth factors and blood vessel formation. Nature 407:242-248. 